\title{
Stability analysis of continuous- time periodic systems via the harmonic analysis
}

\author{
$\operatorname{AUTHOR}(\mathrm{S})$ : \\ Zhou, J; Hagiwara, T; Araki, M
}

\section{CITATION:}

Zhou, J...[et al]. Stability analysis of continuous-time periodic systems via the harmonic analysis. IEEE TRANSACTIONS ON AUTOMATIC CONTROL 2002, 47(2): 292-298

\section{ISSUE DATE:}

2002-02

URL:

http://hdl.handle.net/2433/39971

\section{RIGHT:}

(c)2002 IEEE. Personal use of this material is permitted. However, permission to reprint/republish this material for advertising or promotional purposes or for creating new collective works for resale or redistribution to servers or lists, or to reuse any copyrighted component of this work in other works must be obtained from the IEEE. 


\title{
Stability Analysis of Continuous-Time Periodic Systems Via the Harmonic Analysis
}

Jun Zhou, Tomomichi Hagiwara, and Mituhiko Araki

\begin{abstract}
Asymptotic stability of finite-dimensional linear continuous-time periodic (FDLCP) systems is studied by the harmonic analysis. It is first shown that stability can be examined with what we call the harmonic Lyapunov equation. Another necessary and sufficient stability criterion is developed via this generalized Lyapunov equation, which reduces the stability test into that of an approximate FDLCP model whose transition matrix can be determined explicitly. By extending the Gerschgorin theorem to linear operators on the linear space $l_{2}$, yet another disc-group criterion is derived, which is only sufficient. Stability of the lossy Mathieu equation is analyzed as a numerical example to illustrate the results.
\end{abstract}

Index Terms-Asymptotic stability, continuous-time periodic systems, harmonic analysis, Lyapunov equation.

\section{INTRODUCTION}

The research in finite-dimensional linear continuous-time periodic (FDLCP) systems has been a focus in system analysis and synthesis for a long time [4], [11], [13], [16]. In particular, the stability analysis of such systems is much harder than that of linear time-invariant (LTI) systems, and only some primitive results are available [4], [7]. Roughly speaking, the Floquet theorem seems to be the best results at hand when

Manuscript received December 7, 2000; revised April 18, 2001 and September 15, 2001. Recommended by Associate Editor D. E. Miller.

The authors are with the Department of Electrical Engineering, Kyoto University, Yoshida, Sakyo-ku, Kyoto 606-8501, Japan (e-mail: zhouj@jaguar.kuee.kyoto-u.ac.jp).

Publisher Item Identifier S 0018-9286(02)02068-8. 
dealing with stability of general FDLCP systems [8], [11], in contrast with the more general stability analysis (e.g., [3], [6], and [14]) of sampled-data systems, which are also periodic [1].

This note sheds a new light on the asymptotic stability problem of a class of general FDLCP systems. By the Floquet theorem and the Toeplitz similarity transformation formula [15], [17], a stability criterion based on what we call the harmonic Lyapunov equation is proved in Section III. Our proof is given only through simple matrix algebra so that the existence problem of steady-state solutions of a periodic timevarying Lyapunov differential matrix equation is circumvented completely. The harmonic Lyapunov equation provides some theoretical insight into the stability problem for an FDLCP system and is useful in deriving a necessary and sufficient stability test based on approximate FDLCP modeling in Section IV. In Section V, by extending the Gerschgorin theorem to operators on the linear space $l_{2}$, a disk-group stability criterion is developed.

In the following, with a little abuse, we say $F(t) \in L_{2}[0, h]$ to mean that $F$ is a matrix function, each element of which is $h$-periodic and belongs to $L_{2}[0, h]$ when its domain is restricted to the interval $[0, h]$. Similarly for other function sets defined over $[0, h] . \mathcal{S}^{+}$denotes the set of all strictly positive definite self-adjoint bounded operators on $l_{2}$. $\mathcal{Z}$ is the set of all integers.

\section{PRELIMINARIES}

Consider the FDLCP autonomous system

$$
G: \dot{x}=A(t) x
$$

where $A(t) \in L_{2}[0, h]$. The transition matrix of (1) for the initial time $t_{0}$ is denoted by $\Phi\left(t, t_{0}\right)$.

Theorem 1 (Floquet Theorem [11], [13]): Let $A(t)$ be defined as above. Then the transition matrix $\Phi\left(t, t_{0}\right)$ is continuous with respect to $t$ and can be expressed as $\Phi\left(t, t_{0}\right)=P\left(t, t_{0}\right) e^{Q\left(t-t_{0}\right)}$ where $P\left(t, t_{0}\right)$ is a nonsingular $h$-periodic matrix and $Q$ is a constant matrix. Moreover, the system is asymptotically stable if and only if the eigenvalues of the monodromy matrix, $\Phi\left(h+t_{0}, t_{0}\right)$, are in the open unit disk, or equivalently, the eigenvalues of $Q$ lie in the open left-half plane.

Remark 1: It is difficult to apply the Floquet theorem in general since there exists no general computation formula for the monodromy matrix $\Phi\left(h+t_{0}, t_{0}\right)$ except when the state matrix $A(t)$ is of special properties such as piecewise constant [4], [16] or commutative [11]. Also, $\Phi\left(h+t_{0}, t_{0}\right)$ can be computed by a numerical solution of the corresponding differential equation. In this case, however, an approximation modeling error will be inevitable. To put it another way, this approach amounts to merely testing stability of some approximate model of the given FDLCP system unless the modeling error is taken into account.

Now let us consider the Fourier series expansion $A(t)=$ $\sum_{m=-\infty}^{+\infty} A_{m} e^{j m \omega_{h} t}$ with $\omega_{h}=2 \pi / h$. The Toeplitz transformation on $A(t)$ [15], denoted by $\mathcal{T}\{A(t)\}$, maps $A(t)$ into an infinite-dimensional block Toeplitz operator [15] (or to be more precise, block Laurent operator [5], [20]) of the form

$$
\mathcal{T}\{A(t)\}:=\left[\begin{array}{ccccc}
\ddots & \vdots & \vdots & \vdots & . \\
\cdots & A_{0} & A_{-1} & A_{-2} & \cdots \\
\cdots & A_{1} & A_{0} & A_{-1} & \cdots \\
\cdots & A_{2} & A_{1} & A_{0} & \cdots \\
. & \vdots & \vdots & \vdots & \ddots
\end{array}\right]=: \underline{A} .
$$

To facilitate the statement, we introduce the set

$$
L_{\mathrm{PCD}}[0, h]:=\left\{f(t): \begin{array}{l}
f(t) \text { is piecewise continuous and } \\
\text { differentiable at a.e. } t \in[0, h]
\end{array}\right\}
$$

where PCD stands for piecewise continuous and differentiable. We also define the set $l_{E}:=\left\{\underline{x} \in l_{2}: \underline{E}(j 0) \underline{x} \in l_{2}\right\} \subset l_{2}$ with $\underline{E}(j 0)=$ $\left.\underline{E}(j \varphi)\right|_{\varphi=0}$. Here

$$
\underline{E}(j \varphi)=\operatorname{diag}\left[\ldots, j \varphi_{-2} I, j \varphi_{-1} I, j \varphi_{0} I, j \varphi_{1} I, j \varphi_{2} I, \ldots\right]
$$

where $\varphi_{k}:=\varphi+k \omega_{h}, \varphi \in\left[-\left(\omega_{h} / 2\right),\left(\omega_{h} / 2\right)\right)$ and the $j \varphi_{0} I$-block is at the center of $\underline{E}(j \varphi)$. The infinite-dimensional matrix $\underline{E}(j \varphi)$ is used for the frequency-response-operator definition in [17]. A fact about $l_{E}$ is that $l_{E}$ is dense in $l_{2}$ [17].

Lemma 1: Assume in the system (1) that $A(t)$ belongs to $L_{\mathrm{PCD}}[0, h]$. Then, $l_{E}$ is $\underline{P}$-invariant, $\underline{P}^{-1}$-invariant, $\underline{P}^{*}$-invariant and $\underline{P}^{-*}$-invariant, where $\underline{P}^{-*}:=\left[\underline{P}^{-1}\right]^{*} . \underline{P}$ is invertible on $l_{E}$ and the unique inverse of $\underline{P}$ on $l_{E}$ is $\underline{P}^{-1}$ restricted to $l_{E}$. Also, it holds on $l_{E} \subset l_{2}$ that

$$
\underline{P}(\underline{Q}-\underline{E}(j 0)) \underline{P}^{-1}=\underline{A}-\underline{E}(j 0)
$$

where $Q=\operatorname{diag}[\ldots, Q, Q, Q, \ldots]$ and $\underline{P}=\mathcal{T}\{P(t, 0)\}$.

Proof: A complete proof is given in [17]. Here, we give an outline of the proof. By the Floquet theorem of [11, Th. 6.3.2], we obtain

$$
P(t, 0) Q=A(t) P(t, 0)-\dot{P}(t, 0) .
$$

By the assumption on $A(t)$, the Fourier series expansion of $P(t, 0)$ is absolutely convergent and that of $A(t)$ converges to $A\left(t_{0}\right)$ for a.e. $t_{0} \in[0, h]$. Hence, by the Mertens theorem, it follows that:

$$
\mathcal{T}\{A(t) P(t, 0)\}=\mathcal{T}\{A(t)\} \mathcal{T}\{P(t, 0)\} .
$$

Again by the assumption on $A(t)$, the first-order derivative of $P(t, 0)$ is piecewise continuous and the second-order derivative of $P(t, 0)$ exists a.e. in $[0, h]$. Thus, by [2, p. 106, Th. 3], $\dot{P}(t, 0)$ is given by $\dot{P}(t, 0)=\sum_{m=-\infty}^{+\infty} j m \omega_{h} P_{m} e^{j m \omega_{h} t}$ (a.e.) through the termwise differentiation, where $\left\{P_{m}\right\}_{m=-\infty}^{+\infty}$ is the Fourier coefficients sequence of $P(t, 0)$. Then, by [15], we are led to

$$
\mathcal{T}\{\dot{P}(t, 0)\}=\underline{E}(j 0) \underline{P}-\underline{P} \underline{E}(j 0) .
$$

Now take $\underline{x} \in l_{E}$. Then $\mathcal{T}\{\dot{P}(t, 0)\} \underline{x} \in l_{2}$. Also, $\underline{P} \underline{E}(j 0) \underline{x} \in l_{2}$ since $\underline{E}(j 0) \underline{x} \in l_{2}$ and $\underline{P}$ is bounded on $l_{2}$. It follows that $\underline{E}(j 0) \underline{P} \underline{x} \in$ $l_{2}$, i.e., $l_{E}$ is $\underline{P}$-invariant. Similarly, $l_{E}$ is $\underline{P}^{-1}$-invariant.

Noting that $\underline{P}$ and $\underline{P}^{-1}$ are also mappings on $l_{E}$, it follows that $\underline{P}$ is invertible on $l_{E}$ and the unique inverse of $\underline{P}$ on $l_{E}$ is nothing but $\underline{P}^{-1}$ restricted to $l_{E}$ since $\underline{P}^{-1} \underline{P} \underline{x}=\underline{P} \underline{P}^{-1} \underline{x}=\underline{x}, \forall \underline{x} \in l_{E}$.

From the above arguments, applying the Toeplitz transformation on (4) gives (3).

To see the assertion that $l_{E}$ is $\underline{P}^{*}$-invariant, we note that $\underline{P}^{*}=$ $\mathcal{T}\left\{P^{*}(t, 0)\right\}$ and that $\dot{P}^{*}(t, 0)$ is piecewise continuous and $\ddot{P}^{*}(t, 0)$ exists a.e. in $[0, h]$. These imply that $\mathcal{T}\left\{\dot{P}^{*}(t, 0)\right\}=\underline{E}(j 0) \underline{P^{*}-}$ $\underline{P}^{*} \underline{E}(j 0)$. From this, the assertion follows immediately. Similarly, one can show that $l_{E}$ is $\underline{P}^{-*}$-invariant.

To determine the set of the eigenvalues of $\underline{A}-\underline{E}(j 0)$, let us define $\Lambda=\left\{\lambda(Q)+j m \omega_{h}: m \in \mathcal{Z}\right\}$ where $\lambda(Q)$ denotes the set of the eigenvalues of $Q$.

Lemma 2: Suppose in the system (1) that $A(t)$ belongs to $L_{\mathrm{PCD}}[0, h]$. Then the system is asymptotically stable if and only if the set $\Lambda$ of the eigenvalues of $\underline{Q}-\underline{E}(j 0)$ lies in the open left-half plane. Moreover, $\Lambda=\Lambda_{A}$ where $\Lambda_{A}$ is the set of the eigenvalues of $\underline{A}-\underline{E}(j 0)$.

Proof: From the Floquet theorem, the first assertion follows immediately. For each $\lambda \in \Lambda$, there exists a nonzero $\underline{x} \in l_{E}$ such that $(Q-\underline{E}(j 0)) \underline{x}=\lambda \underline{x}$. Noting that $\underline{P} \underline{x} \in l_{E}$ since $l_{E}$ is $\underline{P}$-invariant, it follows from Lemma 1 that $(\underline{A}-\underline{E}(j 0)) \underline{P} \underline{x}=\underline{P}(Q-\underline{E}(j 0)) \underline{x}=$ $\lambda \underline{P} \underline{x}$. This implies that $\Lambda \subset \Lambda_{A}$ since $\underline{P}$ is invertible on $l_{E}$. Similarly it can be shown that $\Lambda_{A} \subset \Lambda$. 


\section{HARMONIC LYAPUNOV EQUATION OF FDLCP SYSTEMS}

In this section, we establish an infinite-dimensional Lyapunov equation for FDLCP systems. To begin with, we need some discussions on the adjoint operator of the unbounded operator $\underline{A}-\underline{E}(j 0)$ viewed on $l_{E}$, which we denote by $(\underline{A}-\underline{E}(j 0))^{*}$. Since $l_{E}$ is dense in $l_{2}$ [17], it is said that $\underline{A}-\underline{E}(j 0)$ is densely defined on $l_{2}[12$, p. 486].

From [5, p. 290], [20], the domain of $(\underline{A}-\underline{E}(j 0))^{*}$ is

$$
\begin{aligned}
& \mathcal{D}\left\{(\underline{A}-\underline{E}(j 0))^{*}\right\} \\
&=\left\{\underline{y} \in l_{2}: \sup _{0=\underline{x} \in l_{E}} \frac{|\langle(\underline{A}-\underline{E}(j 0)) \underline{x}, \underline{y}\rangle|}{\|\underline{x}\|_{l_{2}}}<\infty\right\} .
\end{aligned}
$$

However, since $\underline{A}$ is bounded on $l_{2}$ (by the assumption that $A(t) \in$ $\left.L_{\mathrm{PCD}}[0, h]\right)$ [17], it follows that:

$$
\begin{aligned}
\mathcal{D}\{(\underline{A}- & \left.\underline{E}(j 0))^{*}\right\} \\
& =\left\{\underline{y} \in l_{2}: \sup _{0=\underline{x} \in l_{E}} \frac{|\langle\underline{E}(j 0) \underline{x}, \underline{y}\rangle|}{\|\underline{x}\|_{l_{2}}}<\infty\right\}=\mathcal{D}\left\{\underline{E}(j 0)^{*}\right\} .
\end{aligned}
$$

On the other hand, by the structure of $\underline{E}(j 0)$, it is obvious that $\underline{E}(j 0)$ is a weighted sum of projections on $l_{2}$. Hence, it follows from [12, Ex. 7.10.1, p. 528] that $\mathcal{D}\left\{\underline{E}(j 0)^{*}\right\}=\mathcal{D}\{\underline{E}(j 0)\}=l_{E}$ and that the matrix representation of $E(j 0)^{*}$ coincides with the usual complex conjugate transpose of that of $E(j 0)$. Summarizing the above arguments, we have verified that $(\underline{A}-\underline{E}(j 0))^{*}$ is also defined on $l_{E}$ and that the matrix representation of $(\underline{A}-\underline{E}(j 0))^{*}$ coincides with the usual complex conjugate transpose of that of $\underline{A}-\underline{E}(j 0)$. Hence, again from $[5$, p. 290], [20], we have

$$
\langle(\underline{A}-\underline{E}(j 0)) \underline{x}, \underline{y}\rangle=\left\langle\underline{x},(\underline{A}-\underline{E}(j 0))^{*} \underline{y}\right\rangle \quad\left(\underline{x}, \underline{y} \in l_{E}\right) .
$$

Now we are in a position to show the following theorem (which is not a special case of the operator-valued Lyapunov equation in [5, Th. I.6.1], [20] since the operator $\underline{A}-\underline{E}(j 0)$ is unbounded).

Theorem 2: Suppose in the system (1) that $A(t)$ belongs to $L_{\mathrm{PCD}}[0, h]$. Then the system is asymptotically stable if and only if for any $\underline{W} \in \mathcal{S}^{+}$, there exists a unique $\underline{V} \in \mathcal{S}^{+}$satisfying

$$
(\underline{A}-\underline{E}(j 0))^{*} \underline{V}+\underline{V}(\underline{A}-\underline{E}(j 0))=-\underline{W}
$$

which is called the (infinite-dimensional) harmonic Lyapunov equation densely defined on $l_{2}$ (or more precisely, defined on the dense subset $l_{E}$ of $l_{2}$ ).

Before giving the proof, we give a remark about the harmonic Lyapunov equation (8). The equation (8) should be viewed as an operator-valued Lyapunov equation densely defined on $l_{2}$. This implies that when we post-multiply $\underline{x} \in l_{2}$ on (8), $\underline{x}$ should belong to $l_{E}$ to guarantee that it makes sense to deal with $(\underline{A}-\underline{E}(j 0))^{*} \underline{V} \underline{x}$ and $\underline{V}(\underline{A}-\underline{E}(j 0)) \underline{x}$ separately and that the inner product is validated in the sense of (7). Now we show that this is indeed the case.

This is equivalent to showing that for the solution $\underline{V}$ of (8), $\underline{V} \underline{x} \in l_{E}$ for any $\underline{x} \in l_{E}$. To this end, take $\underline{x} \in l_{E}$ and post-multiply it on (8). Then, since $\underline{W}, \underline{V}$ and $\underline{A}$ are bounded on $l_{2}$, it follows that $\underline{V}(\underline{A}-$ $\underline{E}(j 0)) \underline{x} \in l_{2}$ and $\underline{W} \underline{x} \in l_{2}$. Now we are led to $(\underline{A}-\underline{E}(j 0))^{*} \underline{V} \underline{x} \in l_{2}$ which, in particular, implies that $-\underline{E}(j 0)^{*} \underline{V} \underline{x} \in l_{2}$. However, since $-\underline{E}(j 0)^{*}=\underline{E}(j 0)$, we can conclude that $\underline{V} \underline{x} \in l_{E}$ as we claimed. The meaning of this remark is that it makes sense to consider the inner product $\left\langle(\underline{A}-\underline{E}(j 0))^{*} \underline{V} \underline{x}, \underline{y}\right\rangle=\langle\underline{V} \underline{x},(\underline{A}-\underline{E}(j 0)) \underline{y}\rangle$ for any $\underline{x}, \underline{y} \in$ $l_{E}$ since $\underline{V} \underline{x} \in l_{E}=\overline{\mathcal{D}}\left((\underline{A}-\underline{E}(j 0))^{*}\right)$.

Proof: Now we give the proof of Theorem 2.

(Sufficiency) Suppose (8) holds for some $\underline{W}, \underline{V} \in \mathcal{S}^{+}$. Let $\lambda$ be an eigenvalue of $\underline{A}-\underline{E}(j 0)$ with an associated eigenvector $\underline{x} \in l_{E} \subset l_{2}$. Then, post-multiplying (8) by $\underline{x}$ and taking the inner product with $\underline{x}$, we obtain $\lambda+\bar{\lambda}=-(\langle\underline{W} \underline{x}, \underline{x}\rangle /\langle\underline{V} \underline{x}, \underline{x}\rangle)<0$ where the inner product is validated from the above discussions. Then, the stability assertion follows from Lemma 2.
(Necessity) Assume that $G$ is asymptotically stable. It must be shown that for any $\underline{W} \in \mathcal{S}^{+}$, there exists a unique operator $\underline{V} \in \mathcal{S}^{+}$ such that (8) holds on $l_{E}$. To this end, we define

$$
\underline{e}(Q, t):=\operatorname{diag}\left[\ldots, e^{\left(Q+j \omega_{h} I\right) t}, e^{Q t}, e^{\left(Q-j \omega_{h} I\right) t}, \ldots\right]
$$

where the $e^{Q t}$-block is at the center of $\underline{e}(Q, t)$. Under the stability assumption, from [4, p. 20], there exist numbers $K>0$ and $\alpha>0$ such that

$$
\operatorname{Re}\{\lambda(Q)\}<-\alpha, \quad\left\|e^{Q t}\right\| \leq K e^{-\alpha t} \quad(\forall t \geq 0) .
$$

From this, it readily follows that $\underline{e}(Q, t)$ is well-defined and uniformly bounded on $l_{2}$ over $t \geq 0$. To see this, we note that $\underline{e}(Q, t)$ is blockdiagonal, and thus

$$
\begin{aligned}
\|\underline{e}(Q, t)\|_{l_{2} / l_{2}} & =\sup _{m \in \mathcal{Z}}\left\|e^{\left(Q+j m \omega_{h} I\right) t}\right\| \\
& =\sup _{m \in \mathcal{Z}}\left\|e^{Q t}\right\| \leq K e^{-\alpha t} \leq K \quad \forall t \geq 0 .
\end{aligned}
$$

Next, we construct the operator

$$
\underline{\hat{V}}:=\int_{0}^{\infty} \underline{e}(Q, \tau)^{*} \underline{\hat{W}} \underline{e}(Q, \tau) d \tau
$$

where $\underline{\hat{W}}=\underline{P^{*}} \underline{W} \underline{P} \in \mathcal{S}^{+}$. It can be shown that $\underline{\hat{V}} \in \mathcal{S}^{+}$and it is a unique solution of

$$
(\underline{Q}-\underline{E}(j 0))^{*} \underline{\hat{V}}+\underline{\hat{V}}(\underline{Q}-\underline{E}(j 0))=-\underline{\hat{W}}
$$

in the elementwise sense (that is, we regard (13) as infinitely many simultaneous equations of finite-dimensional matrices with infinitely many finite-dimensional matrix variables). This can be completed by similar arguments as we do in LTI stable systems. The main difficulties are that the matrices here are infinite-dimensional and order interchanges between infinite-dimensional matrices and infinite integral $\left(\int_{0}^{\infty}\right)$ occurred. These order interchanges are validated since $Q-$ $\underline{E}(j 0)$ and $\underline{e}(Q, t)$ are block-diagonal.

Now repeating the arguments about the adjoint of $\underline{A}-\underline{E}(j 0)$ on $\underline{Q-}$ $\underline{E}(j 0)$, it follows readily that (13) can also be viewed as an operatorvalued (but with infinite-dimensional matrix representation) Lyapunov equation on $l_{E} \subset l_{2}$ and that $l_{E}$ is $\hat{V}$-invariant.

By pre-multiplying $\underline{P}^{-*}\left(=\left[\underline{P}^{-1}\right]^{*}\right)$ and post-multiplying $\underline{P}^{-1}$ on (13) and noting that $l_{E}$ is $\underline{P}^{-1}$-invariant by Lemma 1 , it follows that on $l_{E} \subset l_{2}$

$$
\begin{aligned}
\underline{P}^{-*}(\underline{Q}-\underline{E}(j 0))^{*} \underline{\hat{V}} \underline{P}^{-1}+\underline{P}^{-*} \underline{\hat{V}}(\underline{Q}-\underline{E}(j 0)) \underline{P}^{-1} \\
=-\underline{P}^{-*} \underline{\hat{W}} \underline{P}^{-1} .
\end{aligned}
$$

On the other hand, since $l_{E}$ is $\underline{P}^{-1}-, \underline{\hat{V}}$ - and $\underline{P}^{-*}$-invariant, it is clear that $\underline{P}^{-*} \hat{V} \underline{P}^{-1} \underline{x} \in l_{E}$ if $\underline{x} \in \bar{l}_{E}$. Therefore, it can be claimed that on $l_{E} \subset l_{2}$

$$
\begin{aligned}
& \underline{P}^{-*}(\underline{Q}-\underline{E}(j 0))^{*} \underline{P}^{*} \underline{P}^{-*} \underline{\hat{V}} \underline{P}^{-1} \\
& \quad+\underline{P}^{-*} \underline{\hat{V}} \underline{P}^{-1} \underline{P}(\underline{Q}-\underline{E}(j 0)) \underline{P}^{-1}=-\underline{P}^{-*} \underline{\hat{W}} \underline{P}{ }^{-1}
\end{aligned}
$$

where $\underline{P}^{*} \underline{P}^{-*}=\underline{I}$ and $\underline{P}^{-1} \underline{P}=\underline{I}$ are the identity operators on $l_{E}$ and $l_{2}$, respectively. Since $\underline{P}^{-1}(Q-\underline{E}(j 0)) \underline{P}^{-1}=\underline{A}-\underline{E}(j 0)$ on $l_{E}$ by (3) and $\underline{P}^{-*}(\underline{Q}-\underline{E}(j 0))^{*} \underline{P}^{*}=(\underline{A}-\underline{E}(j 0))^{*}$ from (3), it follows that on $l_{E} \subset l_{2}$

$$
\begin{aligned}
(\underline{A}-\underline{E}(j 0))^{*} \underline{P}^{-*} \hat{\hat{V}} \underline{P}^{-1}+\underline{P}^{-*} \underline{\hat{V}} \underline{P}^{-1}(\underline{A}-\underline{E}(j 0)) \\
=-\underline{P}^{-*} \underline{\hat{W}} \underline{P}^{-1} .
\end{aligned}
$$

Finally, noting that $\underline{P}^{-*} \underline{\hat{W}} \underline{P}^{-1}=\underline{W}$, it follows that $\underline{V}:=$ $\underline{P}^{-*} \hat{V} \underline{P}^{-1} \in \mathcal{S}^{+}$is a unique solution of (8).

Theorem 2 is an operator explanation of asymptotic stability of FDLCP systems. It has been shown [19] that the harmonic Lyapunov equation plays a key role also in developing the trace formula for the $\mathrm{H}_{2}$ norm of FDLCP systems. To demonstrate a further application of the harmonic Lyapunov equation, in our following discussions we 
concentrate our attention on showing that this equation can provide us with some more practical stability tests.

\section{Stability CRITERIon BASEd on AN APPROXIMATE Model}

In this section, we revisit the well-known approximate modeling method [4], [7]. The basic idea is that if we construct an approximate model to the original FDLCP system in some sense such that the transition matrix of this approximate model can be determined explicitly in a closed form (so that this transition matrix knowledge can be used in stability testing of the approximate model), then we are confronted with such a question: under what conditions, can one guarantee the stability of the original FDLCP system by that of the approximate model? The main difficulties in such a stability analysis method include: how to measure the modeling error and how to assess its affect on the stability of the actual system.

Now define the approximate FDLCP model of $G$ by

$$
G_{a}: \dot{y}=A_{a}(t) y
$$

which has the explicit state transition matrix $\Phi_{a}(t, 0)=$ $P_{a}(t, 0) e^{Q_{a} t} . A_{a}(t)$ is the approximate state matrix and $A_{\Delta}(t)$ is the error matrix such that $A(t)=A_{a}(t)+A_{\Delta}(t)$. We assume that $A_{a}(t)$ and $A_{\Delta}(t)$ are $h$-periodic. Obviously, $\underline{A}=\underline{A}_{a}+\underline{A}_{\Delta}$ with $\underline{A}_{a}:=\mathcal{T}\left\{A_{a}(t)\right\}$ and $\underline{A}_{\Delta}:=\mathcal{T}\left\{A_{\Delta}(t)\right\}$. By the Fourier series expansion from $L_{2}[0, h]$ to $l_{2}$, it is can be shown [17] that for $A_{\Delta}(t) \in L_{\mathrm{PCD}}[0, h]$,

$$
\begin{aligned}
\left\|\underline{A}_{\Delta}\right\|_{l_{2} / l_{2}} & =\left\|A_{\Delta}(\cdot)\right\|_{L_{2}[0, h] / L_{2}[0, h]} \\
& =\sup _{t \in[0, h)}\left\|A_{\Delta}(t)\right\|=:\left\|A_{\Delta}(\cdot)\right\| .
\end{aligned}
$$

Based on these preparations, the following theorem gives an answer to the question we posed.

Theorem 3: Suppose $A(t) \in L_{\mathrm{PCD}}[0, h]$ and let $L_{a}[0, h]$ be a dense subset of $L_{\mathrm{PCD}}[0, h]$ in the $L_{\infty}[0, h]$-norm sense (and hence $L_{a}[0, h]$ is dense in $L_{\mathrm{PCD}}[0, h]$ also in the $L_{2}[0, h]$-norm sense). Then the system (1) is asymptotically stable if and only if there exists an approximate $h$-periodic system $G_{a}$ as defined in (14) such that

1) $A_{a}(t) \in L_{a}[0, h]$;

2) $G_{a}$ has the transition matrix $\Phi_{a}(t, 0)=P_{a}(t, 0) e^{Q_{a} t}$ and all the eigenvalues of $Q_{a}$ have negative real parts;

3) for $A_{\Delta}(t)=A(t)-A_{a}(t)$, there exist numbers $K_{a}>0$ and $\alpha>0$ satisfying

$$
\left\{\begin{array}{l}
\left\|e^{Q_{a} t}\right\| \leq K_{a} e^{-\alpha t} \quad(\forall t \geq 0) \\
\sup _{t \in[0, h)}\left\|P_{a}^{-1}(t, 0) A_{\Delta}(t) P_{a}(t, 0)\right\|<\alpha / K_{a}^{2} .
\end{array}\right.
$$

Remark 2: It is clear that numerical computation errors may appear when the stability conditions of Theorem 3 are investigated via certain numerical analysis tools. Apparently, this kind of numerical errors are essentially different from the modeling errors and should be treated as a separate problem in the application of this theorem. In other words, we are isolating the issue of modeling errors from that of numerical computation errors completely in the arguments of Theorem 3.

Remark 3: Theorem 3 shows from the modeling error viewpoint that computing merely the eigenvalues of the corresponding monodromy matrix of an approximate model is not sufficient, theoretically speaking, to check whether or not an FDLCP system is stable however high the approximation accuracy may be. Indeed, as we discussed in Remark 1, any direct but approximate computation of the monodromy matrix $\Phi\left(t_{0}+h, t_{0}\right)$ is insufficient for testing stability of a general FDLCP system unless the modeling error is taken into account. The importance of Theorem 3 lies in that it can ensure stability provided that the approximate model is stable enough in the sense that the condition 3) is satisfied.
Proof: Now we give the proof of Theorem 3.

(Sufficiency) By the condition 2) and Theorem 2, for any $\underline{W}_{a} \in$ $\mathcal{S}^{+}$, the harmonic Lyapunov equation

$$
\left(\underline{A}_{a}-\underline{E}(j 0)\right)^{*} \underline{V}_{a}+\underline{V}_{a}\left(\underline{A}_{a}-\underline{E}(j 0)\right)=-\underline{W}_{a}
$$

has a unique solution $\underline{V}_{a} \in \mathcal{S}^{+}$. In particular, let us take $\underline{W}_{a}=$ $\underline{P}_{a}^{-*} \underline{P}_{a}^{-1} \in \mathcal{S}^{+}$. Then, we have

$$
\underline{V}_{a}=\underline{P}_{a}^{-*}\left\{\int_{0}^{\infty} \underline{e}\left(Q_{a}, \tau\right)^{*} \underline{e}\left(Q_{a}, \tau\right) d \tau\right\} \underline{P}_{a}^{-1} .
$$

On the other hand, (16) can be rewritten as

$$
\begin{aligned}
\left(\underline{A}_{a}+\underline{A}_{\Delta}-\underline{E}(j 0)\right)^{*} & \underline{V}_{a}+\underline{V}_{a}\left(\underline{A}_{a}+\underline{A}_{\Delta}-\underline{E}(j 0)\right) \\
& =-\left(\underline{P}_{a}^{-*} \underline{P}_{a}^{-1}-\underline{A}_{\Delta}^{*} \underline{V}_{a}-\underline{V}_{a} \underline{A}_{\Delta}\right) .
\end{aligned}
$$

Now take $0 \neq \underline{x} \in l_{E} \subset l_{2}$. Then by the well-known Cauchy-Schwarz inequality, we obtain

$$
\begin{aligned}
& \mid\left\langle\underline{A}_{\Delta}^{*}\right.\left.\underline{P}_{a}^{-*}\left\{\int_{0}^{\infty} \underline{e}\left(Q_{a}, \tau\right)^{*} \underline{e}\left(Q_{a}, \tau\right) d \tau\right\} \underline{P}_{a}^{-1} \underline{x}, \underline{x}\right\rangle \mid \\
&=\mid\left\langle\underline{P}_{a}^{*} \underline{A}_{\Delta}^{*} \underline{P}_{a}^{-*}\left\{\int_{0}^{\infty} \underline{e}\left(Q_{a}, \tau\right)^{*} \underline{e}\left(Q_{a}, \tau\right) d \tau\right\}\right. \\
&\left.\cdot \underline{P}_{a}^{-1} \underline{x}, \underline{P}_{a}^{-1} \underline{x}\right\rangle \mid \\
& \leq\left\|\underline{P}_{a}^{-1} \underline{x}\right\|_{l_{2}}^{2}\left\|\underline{P}_{a}^{-1} \underline{A}_{\Delta} \underline{P}_{a}\right\|_{l_{2} / l_{2}} \int_{0}^{\infty} K_{a}^{2} e^{-2 \alpha \tau} d \tau \\
&=\left\|\underline{P}_{a}^{-1} \underline{x}\right\|_{l_{2}}^{2} \sup _{t \in[0, h)}\left\|P_{a}^{-1}(t, 0) A_{\Delta}(t) P_{a}(t, 0)\right\| \frac{K_{a}^{2}}{2 \alpha} \\
&<\frac{1}{2} \| \underline{P}_{a}^{-1} \underline{x}_{l_{2}}^{2}
\end{aligned}
$$

where we used the condition 3 ) and followed a similar derivation as in (15) on the operator $\underline{P}_{a}^{-1} \underline{A}_{\Delta} \underline{P}_{a}$. Also, for any $0 \neq \underline{x} \in l_{E} \subset l_{2}$, $\underline{P}_{a}^{-1} \underline{x} \neq 0$. Summarizing the above arguments, it can be concluded that for any $0 \neq \underline{x} \in l_{E} \subset l_{2},\left\langle\left(\underline{P}_{a}^{-*} \underline{P}_{a}^{-1}-\underline{A}_{\Delta}^{*} \underline{V}_{a}-\underline{V}_{a} \underline{A}_{\Delta}\right) \underline{x}, \underline{x}\right\rangle>0$.

Finally, we confine $\underline{x}$ to be an eigenvector of the operator $\underline{A}_{a}+$ $\underline{A}_{\Delta}-\underline{E}(j 0)(=\underline{A}-\underline{E}(j 0))$ corresponding to an eigenvalue $\lambda$ (note that eigenvectors belong to $l_{E}$ by definition [12, p. 411]. Postmultiplying $\underline{x}$ on (18) and taking the inner product with $\underline{x}$, it follows that $2 \operatorname{Re}(\lambda)\left\langle\underline{V}_{a} \underline{x}, \underline{x}\right\rangle<0$. Noting that $\underline{V}_{a} \in \mathcal{S}^{+}$, this actually says that all the eigenvalues of $\underline{A}-\underline{E}(j 0)$ have negative real parts. Thus the system $G$ is asymptotically stable by Lemma 2 .

(Necessity) Assuming that the system (1) is asymptotically stable, we complete the proof in two steps.

Step 1): First we show that the condition 2) is satisfied. To see this, it is shown that for $t \in[0, h]$

$$
\left\{\begin{array}{l}
\lim _{\left\|A_{\Delta}(\cdot)\right\| \rightarrow 0}\left\|\Phi(t, 0)-\Phi_{a}(t, 0)\right\|=0 \\
\lim _{\left\|A_{\Delta}(\cdot)\right\| \rightarrow 0}\left\|e^{Q_{a} t}-e^{Q t}\right\|=0 .
\end{array}\right.
$$

From (14), we can observe that $\dot{\Phi}_{a}(t, 0)=A(t) \Phi_{a}(t, 0)-$ $A_{\Delta}(t) \Phi_{a}(t, 0)$. According to the variation-of-constants formula [7], it follows that

$$
\begin{aligned}
\Phi_{a}(t, 0)-\Phi(t, 0) & \\
= & -\int_{0}^{t} \Phi(t, \tau) A_{\Delta}(\tau) \Phi_{a}(\tau, 0) d \tau \\
= & -\int_{0}^{t} \Phi(t, \tau) A_{\Delta}(\tau)\left[\Phi_{a}(\tau, 0)-\Phi(\tau, 0)\right] d \tau \\
& -\int_{0}^{t} \Phi(t, \tau) A_{\Delta}(\tau) \Phi(\tau, 0) d \tau .
\end{aligned}
$$


Let $\hat{K}:=\sup _{\tau, t \in[0, h]}\|\Phi(t, \tau)\|<\infty$. Then it follows that for any $t \in[0, h]$

$$
\begin{aligned}
&\left\|\Phi_{a}(t, 0)-\Phi(t, 0)\right\| \leq \hat{K}^{2} h\left\|A_{\Delta}(\cdot)\right\| \\
&+\hat{K}\left\|A_{\Delta}(\cdot)\right\| \int_{0}^{t}\left\|\Phi_{a}(\tau, 0)-\Phi(\tau, 0)\right\| d \tau .
\end{aligned}
$$

Since $L_{\mathrm{PCD}}[0, h]$ consists only of piecewise continuous functions, $\left\|A_{\Delta}(\cdot)\right\|=\sup _{t \in[0, h]}\left\|A_{\Delta}(t)\right\|$ is well-defined regardless of the choice of $L_{a}[0, h]$ and $A_{a}(t)$. Furthermore, by the assumption, $\left\|A_{\Delta}(\cdot)\right\|$ can be made arbitrarily small by a suitable choice of $A_{a}(t)$. Noting also that $\Phi(t, 0)$ and $\Phi_{a}(t, 0)$ are continuous, it follows from Gronwall's Lemma [4], [7] that

$$
\left\|\Phi_{a}(t, 0)-\Phi(t, 0)\right\| \leq \hat{K}^{2} h\left\|A_{\Delta}(\cdot)\right\| e^{K h\left\|A_{\Delta}(\cdot)\right\|}
$$

which clearly says that as $\left\|A_{\Delta}(\cdot)\right\| \rightarrow 0, \Phi_{a}(t, 0) \rightarrow \Phi(t, 0)$ uniformly with respect to $t \in[0, h]$.

To show the second relation of (20), we notice from (22) that $\lim _{\left\|A_{\Delta}(\cdot)\right\| \rightarrow 0} \Phi_{a}(h, 0)=\Phi(h, 0)$ which implies that the eigenvalues of $\Phi_{a}(h, 0)$ tend to those of $\Phi(h, 0)$ as $\left\|A_{\Delta}(\cdot)\right\| \rightarrow 0$. Since $\Phi_{a}(h, 0)$ and $\Phi(h, 0)$ are nonsingular, it follows from [9, Th. 6.4.20] that we can construct a continuous function $\log (\cdot)$ over the set of $\Phi_{a}(h, 0)$ corresponding to sufficiently small $\left\|A_{\Delta}(\cdot)\right\|$ such that $\exp \left(\log \Phi_{a}(h, 0)\right)=\Phi_{a}(h, 0)$. Hence, it follows immediately that:

$$
\lim _{\left\|A_{\Delta}(\cdot)\right\| \rightarrow 0} Q_{a}=Q
$$

which says that the condition 2) is satisfied.

Now considering $\dot{\mu}=Q_{a} \mu$ and $\dot{v}=Q v$ and repeating the arguments of (21) and (22) on these two equations and applying Gronwall's Lemma leads to the second relation of (20).

By similar arguments, it can be asserted that for any $t \in[0, h]$

$$
\left\{\begin{array}{l}
\lim _{\left\|A_{\Delta}(\cdot)\right\| \rightarrow 0}\left\|\Phi_{a}^{-1}(t, 0)-\Phi^{-1}(t, 0)\right\|=0 \\
\lim _{\left\|A_{\Delta}(\cdot)\right\| \rightarrow 0}\left\|e^{-Q_{a} t}-e^{-Q t}\right\|=0 .
\end{array}\right.
$$

Step 2): Now we show that the condition 3) is satisfied. Let us define the set

$$
\mathcal{A}_{\delta}:=\left\{A_{a}(t) \in L_{a}[0, h]: \sup _{t \in[0, h)}\left\|A_{\Delta}(t)\right\| \leq \delta\right\}
$$

with $\delta$ being a constant. Now we further denote the closure of $\mathcal{A}_{\delta}$ by $\overline{\mathcal{A}}_{\delta}$, which is well-defined since $L_{a}[0, h]$ is dense in $L_{\mathrm{PCD}}[0, h]$. Clearly, $\overline{\mathcal{A}}_{\delta}$ is bounded and closed for any fixed $\delta$. From (23) and the strictness of the first inequality in (10), there exists a small enough $\delta>0$ such that for some $\beta>\alpha$, every $A_{a}(t) \in \overline{\mathcal{A}}_{\delta}$ will be $\beta$-stable in the sense that $\operatorname{Re}\left\{\lambda\left(Q_{a}\right)\right\}<-\beta$. Therefore, for each $A_{a}(t) \in \overline{\mathcal{A}}_{\delta}$ there exists a finite number $K_{a}\left(Q_{a}\right)>0$ such that

$$
\left\|e^{Q_{a} t}\right\| \leq K_{a}\left(Q_{a}\right) e^{-\beta t} \quad(\forall t \geq 0) .
$$

In the sequel, we take one such small enough $\delta$. Furthermore, note that

$$
\begin{aligned}
\sup _{t \in[0, h]}\left\|P_{a}(t, 0)\right\| & =\sup _{t \in[0, h]}\left\|\Phi_{a}(t, 0) e^{-Q_{a} t}\right\| \\
& \leq \sup _{t \in[0, h]}\left\|\Phi_{a}(t, 0)\right\| \sup _{t \in[0, h]}\left\|e^{-Q_{a} t}\right\| .
\end{aligned}
$$

Then, by the definition of $\overline{\mathcal{A}}_{\delta}$, (20) and (24), the uniform boundedness of $\sup _{t \in[0, h]}\left\|P_{a}(t, 0)\right\|$ over $\overline{\mathcal{A}}_{\delta}$ follows. Similarly for $\sup _{t \in[0, h]}\left\|P_{a}^{-1}(t, 0)\right\|$.

To see that the condition 3 ) holds, we observe

$$
\begin{aligned}
\mu & :=\sup _{t \in[0, h]}\left\|P_{a}^{-1}(t, 0) A_{\Delta}(t) P_{a}(t, 0)\right\| \\
& \leq \sup _{t \in[0, h]}\left\|P_{a}^{-1}(t, 0)\right\| \sup _{t \in[0, h]}\left\|A_{\Delta}(t)\right\| \sup _{t \in[0, h]}\left\|P_{a}(t, 0)\right\| .
\end{aligned}
$$

By the uniform boundedness of the first and third factors in the right-hand side of (27), $\mu$ can be made arbitrarily small by taking appropriate $A_{a}(t) \in \mathcal{A}_{\delta}$. Therefore, the proof is completed if we show that the first requirement in the condition 3) can be satisfied with fixed $K_{a}>0$ and $\alpha>0$ independent of $A_{a}(t)$. More specifically, we show that there exists $K_{a}>0$ such that

$$
\left\|e^{Q_{a} t}\right\| \leq K_{a} e^{-\alpha t} \quad\left(\forall t \geq 0, \forall A_{a}(t) \in \mathcal{A}_{\delta} \subset \overline{\mathcal{A}}_{\delta}\right)
$$

where $\alpha$ is as given in (10). However, this can be completed by similar arguments to those in Step 1 [see also (25)] and the well-known Heine-Borel finite-covering theorem. The details are omitted due to the limited space. A complete proof can be found in [18].

Note that the sufficiency proof does not rely on the assumption that $L_{a}[0, h]$ is dense in $L_{\mathrm{PCD}}[0, h]$. This implies that $A_{a}(t)$ can be any approximate model as long as $A_{a}(t) \in L_{\mathrm{PCD}}[0, h]$ and its transition matrix can be determined by some approach. Indeed, [7] gave a similar but sufficient stability test by constant approximation, which is derived by Gronwall's Lemma [4], [7].

In spite of a large freedom in choosing $A_{a}(t)$, however, trial-anderror is needed in choosing $A_{a}(t)$ when one attempts to show the stability of a given FDLCP system with Theorem 3 . In such a case, it is sensible to consider the dense subset $L_{a}[0, h]$ from which $A_{a}(t)$ is taken, and a reasonable candidate for $L_{a}[0, h]$ is the set of all piecewise constant functions, which is denoted by $L_{\mathrm{pc}}[0, h]$. It is well-known [4], [16] that for any $A_{a}(t) \in L_{\mathrm{pc}}[0, h]$, the transition matrix $\Phi_{a}(t, 0)$ can be computed explicitly, so that the condition 3 ) of Theorem 3 is easy to check. The necessity of Theorem 3 ensures that it is always possible to find an approximate model in $L_{\mathrm{pc}}[0, h]$ to satisfy the conditions by letting $\left\|A_{\Delta}(\cdot)\right\| \rightarrow 0$ when the system is stable.

Also it should be pointed out that the sufficiency part can be verified by using the variation-of-constants formula and Gronwall's Lemma after some trivial modifications on the condition 3). However, the proof given via the harmonic Lyapunov equation is an independent alternative method, which explains the asymptotic stability of a class of FDLCP systems from the operator-theoretic viewpoint instead of the asymptotic analysis of differential equations.

\section{GERSCHGORIN STABILITY CRITERION}

In this section, we discuss a sufficient stability test by extending the Gerschgorin theorem [10] to operators on $l_{2}$. Now introduce the set $L_{\mathrm{CPCD}}[0, h]$ consisting of all functions that are continuous and whose first-order derivatives are piecewise continuous over $[0, h]$. Obviously, $L_{\mathrm{CPCD}}[0, h] \subset L_{\mathrm{PCD}}[0, h]$ so that Lemmas 1 and 2 apply if $A(t) \in$ $L_{\mathrm{CPCD}}[0, h]$. Now we show the extended Gerschgorin criterion.

Theorem 4: Suppose that the $n \times n$ state matrix $A(t)$ belongs to $L_{\mathrm{CPCD}}[0, h]$ and $\left\{A_{m}\right\}_{m=-\infty}^{+\infty}$ is the corresponding Fourier coefficients sequence of $A(t)$. Then the system (1) is asymptotically stable if the disc-group $\mathcal{D}_{0}:=\bigcup_{k=1}^{n} \mathcal{D}_{0 k}$ lies in the open left-half plane. Here

$$
\mathcal{D}_{0 k}:=\left\{z \in \mathcal{C}:\left|z-a_{0 k k}\right| \leq \Delta_{k}\right\} \quad k=1,2, \ldots, n
$$

with $\Delta_{k}=\sum_{i=1}^{n} \sum_{m=-\infty}^{+\infty}\left|a_{m k i}\right|-\left|a_{0 k k}\right|$ where $a_{m k i}$ is the $(k, i)$ th entry of the matrix $A_{m}$.

Furthermore, suppose that there are $m(<n)$ discs $\mathcal{D}_{0 i_{1}}, \mathcal{D}_{0 i_{2}}, \ldots$, $\mathcal{D}_{0 i_{m}}$ such that $\mathcal{D}_{0}^{\prime}+j l \omega_{h}$ and $\mathcal{D}_{0}^{\prime \prime}$, with $\mathcal{D}_{0}^{\prime}:=\bigcup_{k=i_{1}, \ldots, i_{m}} \mathcal{D}_{0 k}$ and $\mathcal{D}_{0}^{\prime \prime}:=\bigcup_{k=i_{1}, \ldots, i_{m}} \mathcal{D}_{0 k}$, are disjoint for all $l \in \mathcal{Z}$. Then, the system (1) is unstable if either $\mathcal{D}_{0}^{\prime}$ or $\mathcal{D}_{0}^{\prime \prime}$ lies in the closed right-half plane.

Proof: Let $\lambda$ be an eigenvalue of $\underline{A}-\underline{E}(j 0)$ with $\underline{x} \in l_{E} \subset l_{2}$ being an associated eigenvector. Then $(\underline{A}-\underline{E}(j 0)) \underline{x}=\lambda \underline{x}$. Now denote $\underline{x}=\left[\ldots, x_{-1}, x_{0}, x_{1}, \ldots\right]^{T}$ where $x_{i}$ is a scalar, and let $\left|x_{s}\right|=\max _{m \in \mathcal{Z}}\left|x_{m}\right|>0$ which can be attained at a finite $s$ since $\underline{x} \in l_{2}$. Using similar arguments to [10], one can show that the eigenvalues of $\underline{A}-\underline{E}(j 0)$ lie in the region $\bigcup_{k=1}^{n} \bigcup_{l=-\infty}^{+\infty} \mathcal{D}_{l k}$ where

$$
\mathcal{D}_{l k}=\left\{z \in \mathcal{C}:\left|z-a_{0 k k}+j l \omega_{h}\right| \leq \Delta_{k}\right\}, \quad l \in \mathcal{Z}
$$

with $k=1,2, \ldots, n$. However, by the definition of $\mathcal{D}_{l k}$, it follows that for each $k, \mathcal{D}_{l k}=\mathcal{D}_{m k}+j(l-m) \omega_{h}$ in the pointwise sense 


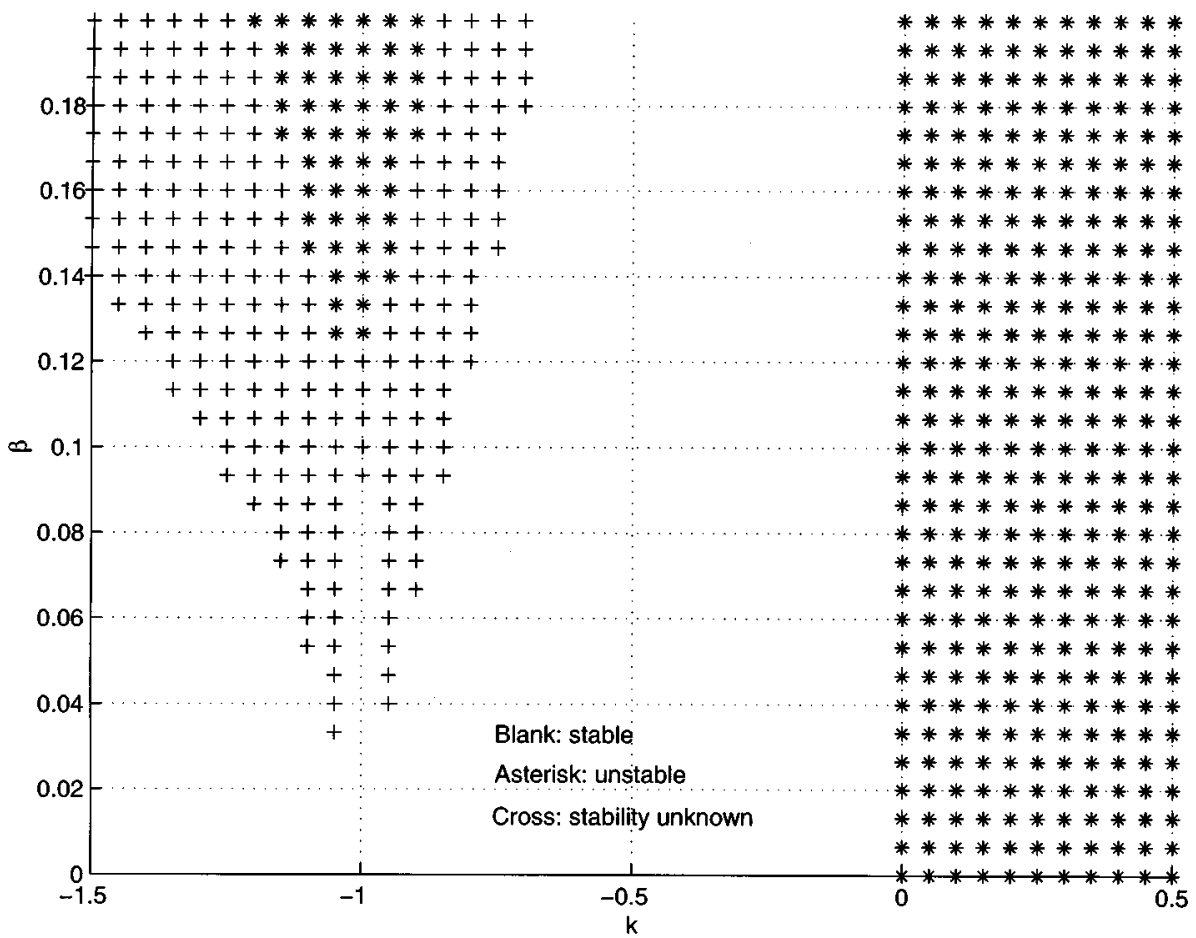

(a)

00000000000000000000000000000000000000000 \$0000000000000000000000000000000000000000 \$0000000000000000000000000000000000000000 0.18400 .0000 .0000 .000000000000000000000 .000000000 00000000000000000000000000000000000000000 $\$ 0000000000000000000000000000000000000000$ 0.1600000000000000000000000000000000000000000 00000000000000000000000000000000000000000 \$0000000000000000000000000000000000000000 0.1440000000000000000000000000000000 .000000000 $\$ 0000000000000000000000000000000000000000$ $\$ 000000000000000000000000000000000000000$

$0.12 \not 000000000000000000000000000.0 \cdots 0.00000000 .0$ S0000000000000000000000000000 00000000000 $\$ 0000000000000000000000000000$

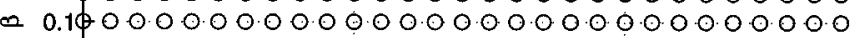
$\$ 0000000000000000000000000000$ $\$ 000000000000000000000000000$

0.084000000000000000000000000000 $\$ 00000000000000000000000000$ $\$ 00000000000000000000000000$

00000000000 00.000000 .00 .0 00000000000 00000000000 00000000.000 00000000000

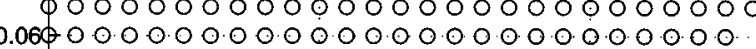
00000000000 ....... 000000000000 00000000000

0.040000000000000000000 \$0 00000000\%000

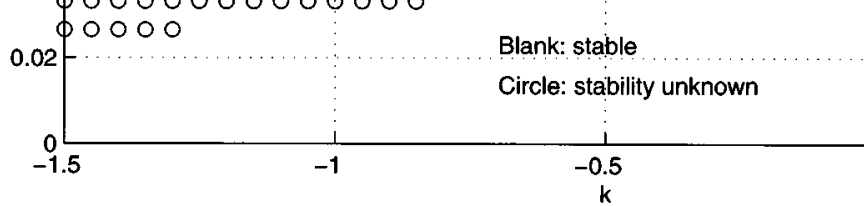
00000000000 00000000000 00000000000 00000000000 00000000000 00000000000 00000000000 00000000000

(b)

Fig. 1. Stable coefficients areas (blank: $G$ stable; asterisk: $G_{a}$ unstable; cross: $G_{a}$ stable but stability of $G$ unknown; circle: stability of $G$ unknown by Gerschgorin criterion).

$(\forall l, m \in \mathcal{Z})$. Hence, if for some $l \in \mathcal{Z}$, the discs $\bigcup_{k=1}^{n} \mathcal{D}_{l k}$ lie in the open left-half plane, then so do all the other discs. This gives the first assertion. The assumption $A(t) \in L_{\mathrm{CPCD}}[0, h]$ ensures by Theorem 2 [2, p. 104] that the disc-group is meaningful in the sense that $\Delta_{k}<\infty$, $\forall k=1,2, \ldots, n$.

To see the second part, let us define $A(\rho, t)=D+\rho(A(t)-D)$ with $\rho$ being a constant in the interval $[0,1]$ and $D=$ $\operatorname{diag}\left[a_{011}, a_{022}, \ldots, a_{0 n n}\right]$. It is clear that for each $\rho \in[0,1]$, $A(\rho, t) \in L_{\mathrm{CPCD}}[0, h]$. Hence, by using Lemma 2 and following similar arguments to those about (23), it is not hard to see that the eigenvalues of the operator $\underline{A}(\rho)-\underline{E}(j 0)$ are continuous with respect to $\rho$.

Now, we define the discs

$$
\mathcal{D}_{l k}(\rho)=\left\{z \in \mathcal{C}:\left|z-a_{0 k k}+j l \omega_{h}\right| \leq \rho \Delta_{k}\right\}, \quad l \in \mathcal{Z}
$$

with $k=1,2, \ldots, n$. From this definition, it is easy to see that $\mathcal{D}_{l k}(\rho) \subset \mathcal{D}_{l k}, \forall \rho \in[0,1], l \in \mathcal{Z}$ and $k=$ 
$1,2, \ldots, n$. From the assumptions about $\mathcal{D}_{0}^{\prime}$ and $\mathcal{D}_{0}^{\prime \prime}$, it follows readily that either $\mathcal{D}^{\prime}(\rho):=\bigcup_{l=-\infty}^{+\infty} \bigcup_{k=i_{1}, \ldots, i_{m}} \mathcal{D}_{l k}(\rho)$ or $\mathcal{D}^{\prime \prime}(\rho):=\bigcup_{l=-\infty}^{+\infty} \bigcup_{k=i_{1}, \ldots, i_{m}} \mathcal{D}_{l k}(\rho)$ lies in the closed right-half plane. On the other hand, the first assertion says that for any $\rho \in[0,1]$, the eigenvalues of $\underline{A}(\rho)-\underline{E}(j 0)$ lie in $\mathcal{D}(\rho):=\bigcup_{k=1}^{n} \bigcup_{l=-\infty}^{+\infty} \mathcal{D}_{l k}(\rho)=\mathcal{D}^{\prime}(\rho) \bigcup \mathcal{D}^{\prime \prime}(\rho)$. Then the second assertion follows from Lemma 2 by letting $\rho=1$, if we show that for any $\rho \in[0,1]$, both $\mathcal{D}^{\prime}(\rho)$ and $\mathcal{D}^{\prime \prime}(\rho)$ contain at least one eigenvalue of the operator $\underline{A}(\rho)-\underline{E}(j 0)$, and thus at least one eigenvalue of $\underline{A}(\rho)-\underline{E}(j 0)$ has nonnegative real part.

To see this, let $\rho=0$ and note that the eigenvalues of $\underline{A}(0)-\underline{E}(j 0)$ are $a_{011}+j l \omega_{h}, a_{022}+j l \omega_{h}, \ldots, a_{0 n n}+j l \omega_{h}, l \in \mathcal{Z}$, which are the centers of the $\operatorname{discs} \mathcal{D}_{l k}(\rho), l \in \mathcal{Z}, \forall \rho \in[0,1]$. By the continuity of the eigenvalues of $\underline{A}(\rho)-\underline{E}(j 0)$ with respect to $\rho$ and the fact that $\mathcal{D}^{\prime}(\rho)$ and $\mathcal{D}^{\prime \prime}(\rho)$ are disjoint, the desired assertion follows.

\section{Stability TeSt of the Lossy Mathieu EQUation}

Now we consider the stability problem of the lossy Mathieu differential equation by using Theorem 3 and the Gerschgorin criterion. The state matrix is given by

$$
A(t)=\left[\begin{array}{cc}
0 & 1 \\
k\left(1-2 \beta \cos \omega_{h} t\right) & -2 \xi
\end{array}\right]
$$

with $\omega_{h}=2$ (i.e., $h=\pi$ ), $\xi=0.06$ and $k$ and $\beta$ being parameters. First we consider to test stability of $A(t)$ by Theorem 3 . To construct an approximate model for each pair of the parameters $(k, \beta)$, we divide the period $h$ into $N_{a}=80$ subintervals of the same length, during each of which $A(t)$ is approximated by a constant matrix. Then, we can easily compute the monodromy matrix $\Phi_{a}(h, 0)$ by matrix exponentiations, as well as $Q_{a}$ by taking a matrix logarithm. To this constant matrix $Q_{a}$, a pair of numbers $K_{a}>0$ and $\alpha>0$ can be found by working on the Jordan canonical form of $Q_{a}:=T_{a} J_{a} T_{a}^{-1}$ and the transition matrix $e^{Q_{a} t}=T_{a} e^{J_{a} t} T_{a}^{-1}$ such that the first inequality of the condition 3) of Theorem 3 is satisfied. We further take $N_{c}=50$ points equitably distributed on each subinterval, and compute the periodic portion $P_{a}(t, 0)$ on each of these $N_{a} N_{c}$ points, which is again carried out by matrix exponentiations since $A_{a}(t)$ is piecewise constant and $Q_{a}$ is already known. Then the second inequality of the condition 3 ) is tested point-by-point on all the $N_{a} N_{c}$ points. Fig. 1(a) is the computation results, in which the blank area is the parameter range when the approximate models used and the original systems are stable, the asterisks $(* \mathrm{~s})$ indicate the parameter range corresponding to unstable approximate models, while at the area marked by crosses $(+s)$ the approximate models are stable but the condition 3 ) is not satisfied for the above $K_{a}$ s and $\alpha$ s.

We can also utilize the Gerschgorin criterion. However, since the structure of $A(t)$ prevents us from applying the criterion effectively, it is necessary to introduce a similarity transformation on $A(t)$ so that the "DC part" becomes diagonal. The computation results are given in Fig. 1(b), in which the blank area is the parameter range where the original systems are stable, while the area marked by circles (os) indicates the parameter range where stability of the original system is uncertain from the extended Gerschgorin criterion, i.e., Theorem 4.

The problem if an approximate model can be found (not only exists), in a finite number of steps, to determine if the original system is stable or unstable, is also worth considering. It is easy to see that this needs an instability criterion to figure out instability cases based on approximate modeling. Unfortunately, however, this kind of criteria still remain as open problems.

\section{CONCLUSION}

In this note, the asymptotic stability problem of a class of general FDLCP systems is studied. Through the harmonic analysis, the Lyapunov equation is recovered in an LTI fashion as an operator-valued Lyapunov equation densely defined on the linear space $l_{2}$, which does help to derive a necessary and sufficient stability criterion via approximate models. The latter is applicable since we can take approximate models whose transition matrix can be explicitly computed. This latter result can be further simplified by introducing upper bounds about the periodic portion of the transition matrix of the approximate model so that the stability conditions rely only on the constant portion knowledge. This improved result will be reported in another paper. The Gerschgorin stability criterion, which is sufficient, is developed by a simple generalization of the finite-dimensional version to operators on the linear space $l_{2}$.

The harmonic Lyapunov equation plays a key role in developing the trace formula for the $\mathrm{H}_{2}$ norm of FDLCP systems, which is a topic of our current study [19] relevant to periodic systems.

\section{REFERENCES}

[1] T. Chen and B. A. Francis, Optimal Sampled-Data Control Systems, 1995.

[2] R. V. Churchill, Fourier Series and Boundary Value Problems, 2nd ed. New York: McGraw-Hill, 1963.

[3] G. Dullerud and K. Glover, "Robust stabilization of sampled-data systems to structured LTI perturbations," IEEE Trans. Automat. Contr., vol. 38, pp. 1497-1507, Oct. 1993.

[4] M. Farkas, Periodic Motions. New York: Springer-Verlag, 1994.

[5] I. Gohberg, S. Goldberg, and M. A. Kaashoek, Classes of Linear Operators. Boston, MA: Birkhäuser, 1990, vol. I.

[6] T. Hagiwara and M. Araki, "Absolute stability of sampled-data systems with a sector nonlinearity," Syst. Control Lett., vol. 27, no. 5, pp. 293-304, 1996.

[7] A. Halanay, Differential Equations Stability, Oscillations, Time Lags.. New York: Academic, 1966.

[8] S. R. Hall and N. M. Wereley, "Generalized Nyquist criterion for linear time systems," in Proc. Amer. Contol Conf., San Diego, CA, 1990, pp. $1518-1525$.

[9] R. A. Horn and C. R. Johnson, Topics in Matrix Analysis. . Cambridge, U.K.: Cambridge Univ. Press, 1991.

[10] P. Lancaster and M. Tismenetsky, The Theory of Matrices., 2nd ed. New York: Academic, 1985.

[11] D. L. Lukes, Differential Equations Classical to Controlled. New York: Academic, 1982.

[12] A. W. Naylor and G. R. Sell, Linear Operator Theory in Engineering and Science. New York: Springer-Verlag, 1982.

[13] G. D. Nicolao, G. F. Trecate, and S. Pinzon, "Zeros of continuous-time linear periodic systems," Automatica, vol. 34, no. 12, pp. 1651-1655, 1998.

[14] N. Sivashankar and P. P. Khargonekar, "Robust stability and performance analysis of sampled-data system," IEEE Trans. Automat. Contr., vol. 38, pp. 58-69, Jan. 1993.

[15] N. M. Wereley, "Analysis and control of linear periodically time varying systems," Ph.D. dissertation, Dept. of Aeronautics and Astronautics, M.I.T., 1990.

[16] J. L. Willems, Stability Theory of Dynamical Systems. Camden, NJ: Nelson, 1970.

[17] J. Zhou and T. Hagiwara, "Existence conditions and properties of frequency response operators of continuous-time periodic systems," SIAM J. Control Optim., to be published.

[18] J. Zhou, T. Hagiwara, and M. Araki, "Stability analysis of continuous-time periodic systems via the harmonic analysis," Automatic Control Engineering Group, Dept. Electrical Engineering, Kyoto Univ., Kyoto, Japan, Tech. Rep., no. 00-06, 2000.

[19] - "Trace formulas for the $H_{2}$ norm of linear continuous-time periodic systems," in Proc. IFAC Workshop Periodic Control Systems, Italy, 2001, pp. 3-8.

[20] I. Gohberg, S. Goldberg, and M. A. Kaashoek, Classes of Linear Operators. Boston, MA: Birkhäuser, 1993, vol. II. 